EPJ Web of Conferences 71, 00049 (2014)

DOI: 10.1051/epjconf/20147100049

(C) Owned by the authors, published by EDP Sciences, 2014

\title{
Recent QCD results from ATLAS
}

\author{
Claudia Glasman ${ }^{1, a}$ (On behalf of the ATLAS Collaboration) \\ ${ }^{1}$ Universidad Autónoma de Madrid, Spain
}

\begin{abstract}
The ATLAS collaboration has performed studies of a wide range of QCD phenomena. Recent soft-QCD measurements include studies of hadronic event shapes and double-parton scattering. Differential measurements of inclusive-jet production provide stringent tests of higher-order QCD predictions and provide input for the determination of the parton density functions. Jet-multiplicity ratios are sensitive to the strong coupling constant and have reduced sensitivity to the uncertainties due to parton distribution functions. Measurements of the inclusive isolated-photon, photon plus jet and diphoton differential cross sections provide a direct probe of short-distance physics, complementary to that from measurements of jets or vector-bosons, and are sensitive to the gluon density of the proton.
\end{abstract}

\section{Introduction}

QCD processes are dominant in hadron colliders and, beyond their intrinsic interest, they constitute the largest background to searches for new physics; thus, a good understanding of these processes is necessary. The jet-production cross section in QCD is given by the convolution of the proton parton density functions (PDFs) and the partonic cross sections; therefore, measurements of jet cross sections provide tests of perturbative QCD (pQCD), determination of the parameters of the theory, such as the strong coupling constant $\left(\alpha_{s}\right)$, constraints of the PDFs and also tuning of the Monte Carlo (MC) models. The lowest-order processes in jet production at LHC are $O\left(\alpha_{s}^{2}\right)$. Multiparton interactions and the soft-underlying event are also intrinsic effects of QCD in hadron-hadron collisions.

Another topic of interest in hadron collisions is the production of prompt photons. Measurements of the production of high transverse momentum $\left(p_{\mathrm{T}}\right)$ photons in hadron colliders also provide tests of pQCD but in a cleaner reaction than jet production; contraints of the proton PDFs are also possible. The study of the production of photons via QCD interactions provides an important input to understand the background to other processes, such as Higgs studies and searches beyond the Standard Model. Prompt photons are produced via two main mechanisms, the so-called direct-photon production, in which the final-state photon is produced directly in the hard interaction, and the fragmentation process, in which the photon arises from the fragmentation of a coloured high $p_{\mathrm{T}}$ parton.

\section{Charged-particle event shapes}

The soft-underlying event can be studied via the measurement of hadronic event shapes. Event-shape observables were used originally to make the first observations of events containing jets in hadron

a e-mail: claudia.glasman@ @ern.ch 
EPJ Web of Conferences

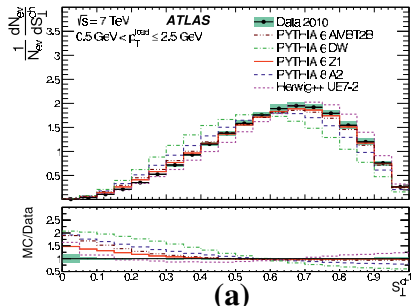

(a)

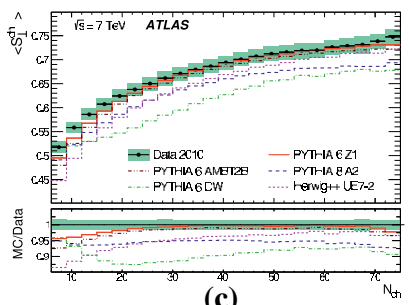

(c)

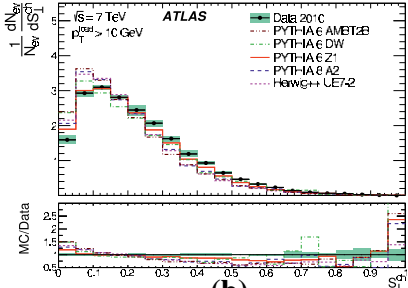

(b)

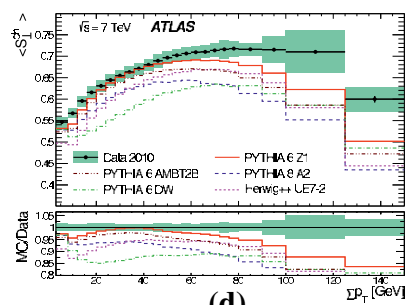

(d)

Figure 1. Transverse sphericity for (a) $0.5<p_{\mathrm{T}}^{\text {lead }} \leq 2.5 \mathrm{GeV}$ and (b) $p_{\mathrm{T}}^{\text {lead }}>10 \mathrm{GeV}$. Mean transverse sphericity as functions of (c) $N_{\mathrm{ch}}$ and (b) $\sum p_{\mathrm{T}}$. [1]

collisions. Observables such as thrust and sphericity are sensitive to hadronisation effects and to the details of the energy flow in the final state and, thus, they are also useful to characterize the properties of the underlying event and other non-perturbative effects. The ATLAS Collaboration has measured several event-shape observables [1] for charged particles with $p_{\mathrm{T}}>0.5 \mathrm{GeV}$ and pseudorapidity $|\eta|<2.5$. Figures $1 \mathrm{a}$ and $1 \mathrm{~b}$ show the distributions as functions of the transverse sphericity for charged particles in different regions of $p_{\mathrm{T}}$ of the leading particle. The transverse sphericity is expected to take values close to 1 for isotropic events and close to 0 for events containing jets. At low $p_{\mathrm{T}}$ (Fig. 1a), the distribution for the data has a high mean value, whereas at high $p_{\mathrm{T}}$ (Fig. 1b), the measured mean value is much smaller. Figures $1 \mathrm{c}$ and $1 \mathrm{~d}$ show the mean values of the transverse sphericity as functions of the number of charged particles and $\sum p_{\mathrm{T}}$, respectively; the measured distribution shown in Fig. 1d shows a decrease at large values of $\sum p_{\mathrm{T}}$ which can be interpreted as the emergence of jets. Various MC models, based on different tunes, are compared to the data in these figures. In particular, the predictions of PYTHIA6-tune Z1, tuned to LHC data, are closest to the data, whereas those of PYTHIA6-tune DW, tuned to Tevatron data, are furthest from the data. There are still regions of phase space in which none of the available tunes describe the data; therefore, these measurements can be used to improve further the models.

\section{Double-parton scattering}

Double-parton interactions have been investigated by ATLAS in $W$ plus two-jet events [2] using an integrated luminosity of $\mathcal{L}=36 \mathrm{pb}^{-1}$; the $W$ boson was identified in the leptonic channel. These events provide a handle to distinguish between direct interactions from those events which proceed via a double-parton interaction; distributions such as $\Delta_{\text {jets }}^{\mathrm{n}}=\left(\left|\vec{p}_{\mathrm{T}}^{\mathrm{J}_{1}}+\vec{p}_{\mathrm{T}}^{\mathrm{J}_{2}}\right|\right) /\left(\left|\vec{p}_{\mathrm{T}}^{\mathrm{J}_{1}}\right|+\left|\vec{p}_{\mathrm{T}}^{\mathrm{J}_{2}}\right|\right)$ (see Fig. 2a) and $\Delta_{\text {jets }}=\left|\vec{p}_{\mathrm{T}}^{J_{1}}+\vec{p}_{\mathrm{T}}^{J_{2}}\right|$ (see Fig. 2b) have different behaviour depending on the process. The fraction of events with a double-parton interaction, $f_{\mathrm{DP}}$, was extracted from these distributions using a fit to the data based on a template from the MC prediction for the direct interaction and a template based on the data for the hard double-parton interactions. The value obtained from the fit to the measured $\Delta_{\text {jets }}^{\mathrm{n}}$ distribution is $f_{\mathrm{DP}}=0.08 \pm 0.01$ (stat.) \pm 0.02 (syst.). This fraction is directly related to 

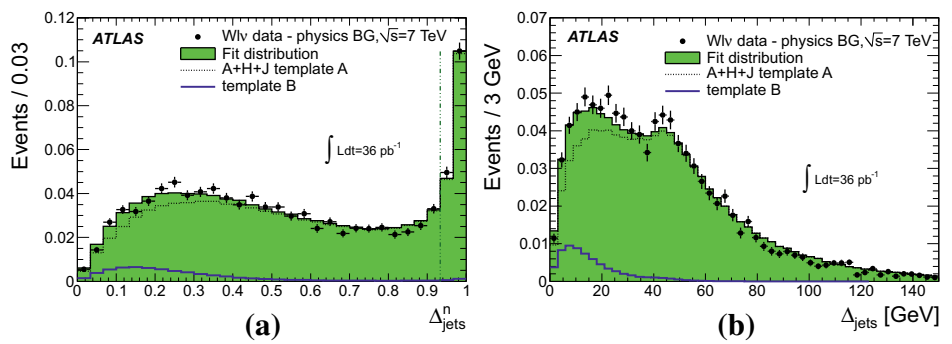

(b)

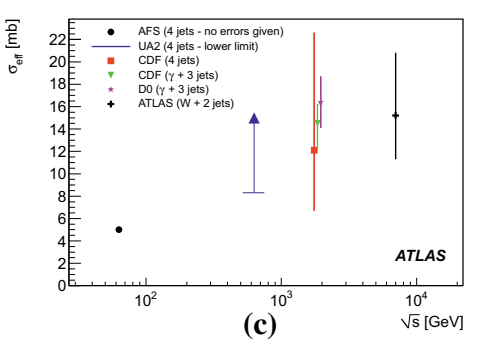

(c)

Figure 2. Distributions as functions of (a) $\Delta_{\text {jets }}^{\mathrm{n}}$ and (b) $\Delta_{\text {jets }}$ for $W$ plus two-jet events. (c) Extracted values of $\sigma_{\text {eff }}$ as a function of the centre-of-mass energy from ATLAS (cross) and other experiments. [2]

the effective area parameter, $\sigma_{\text {eff }}$, which can be used to compare with other experiments. A value of $\sigma_{\text {eff }}(7 \mathrm{TeV})=15 \pm 3$ (stat. $)_{-3}^{+5}$ (syst.) mb was extracted from these measurements. This result is shown in Fig. 2c together with the results from other experiments at lower centre-of-mass energies and found to be consistent.

\section{Inclusive-jet cross sections}

Measurements of inclusive-jet cross sections were performed by ATLAS using the data collected at $2.76 \mathrm{TeV}$, using an integrated luminosity of $\mathcal{L}=0.2 \mathrm{pb}^{-1}$ [3]. The measurements were performed using the anti- $k_{t}$ algorithm for two different jet radii, $R=0.4$ and 0.6 . The jets were selected with $p_{\mathrm{T}}>$ $20 \mathrm{GeV}$. Figure $3 \mathrm{a}$ shows the differential inclusive-jet cross sections as functions of $p_{\mathrm{T}}$ in different regions of jet rapidity $(y)$ for $R=0.6$. Values of $p_{\mathrm{T}}$ up to $430 \mathrm{GeV}$ are accessible with the available luminosity. The $p_{\mathrm{T}}$ spectrum becomes harder as the $y$ is more central. The experimental uncertainties are between $10-30 \%$ in the central $y$ region and higher in other regions and are dominated by the jet energy scale in all $y$ regions. The next-to-leading-order (NLO) QCD predictions from NLOJET++ are compared to the data in Fig. 3a. The calculations were performed with renormalisation $\left(\mu_{R}\right)$ and factorisation $\left(\mu_{F}\right)$ scales set to the maximum jet $p_{\mathrm{T}}$ in each $y$ region and using several proton PDFs, namely CT10, MSTW2008, NNPDF 2.1, HERAPDF 1.5 and ABM 11 NLO; the calculations were corrected for non-perturbative effects. The NLO QCD predictions describe the data within the uncertainties, as seen in Fig. 3b, which shows the ratio of the data and the theory. The yellow bands in this figure represent the theoretical uncertainties, which amount to $5-15 \%$ for central rapidities but increase up to $80 \%$ in other regions of phase space. The uncertainty due to the PDFs is dominant in some regions of phase space. Also in these regions, differences between the predictions from different PDFs are observed; thus, these data have the potential to constrain the proton PDFs.

A comparison with similar ATLAS measurements at $7 \mathrm{TeV}$ was performed [3] via the ratio of the dimensionless scale-invariant cross sections, $F\left(y, x_{\mathrm{T}}, \sqrt{s}\right)=(s / 8 \pi) x_{\mathrm{T}}^{3}\left(d^{2} \sigma / d x_{\mathrm{T}} d y\right)$, of the 2.76 to the $7 \mathrm{TeV}$ data. This ratio was measured as a function of the dimensionless variable $x_{\mathrm{T}}=2 p_{\mathrm{T}} / \sqrt{s}$ in different $y$ regions. The ratio $\rho\left(y, x_{\mathrm{T}}\right)=F\left(y, x_{\mathrm{T}}, 2.76 \mathrm{TeV}\right) / F\left(y, x_{\mathrm{T}}, 7 \mathrm{TeV}\right)$ is shown in Fig. 3c. These measurements provide more stringent tests of pQCD since the correlated uncertainties cancel in the ratio. The experimental uncertainties are $5-20 \%$ and the theoretical uncertainties are reduced to below $2 \%$ in the central region and to $2-10 \%$ in the most-forward region. There is a good description of the data by the NLO predictions. A value between 1.1 and 1.5 is measured for the ratio; such a constant behaviour is expected from the scale evolution of the PDFs and $\alpha_{s}$.

The inclusive-jet cross sections are sensitive to the proton PDFs. The data sets corresponding to $\sqrt{s}=2.76$ and $7 \mathrm{TeV}$ have been included, together with HERA data, in a NLO QCD fit to extract 


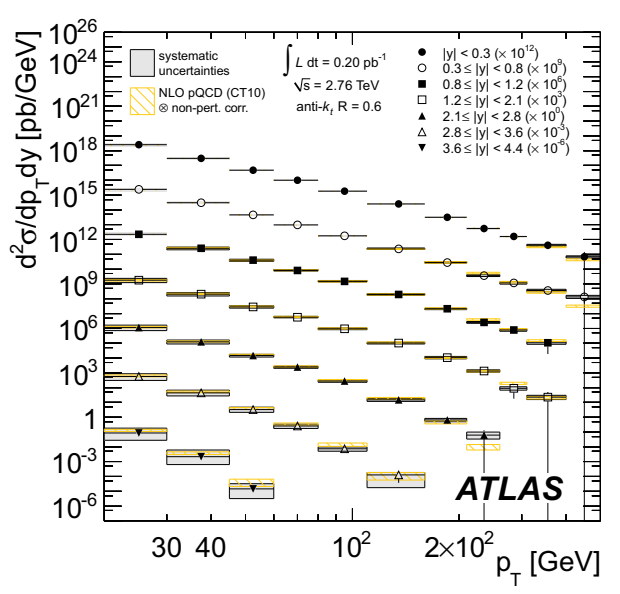

(a)
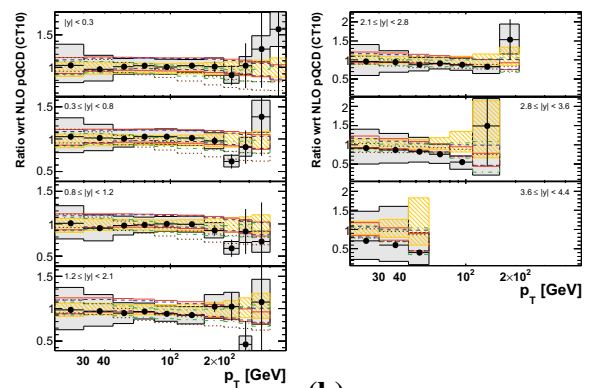

ATLAS

$\int L d t=0.20 \mathrm{pb}^{-1}$ $\sqrt{s}=2.76 \mathrm{TeV}$
anti- $k_{t} R=0.6$

Data with
-- statistical

uncertainty NLO PQCD $\otimes$ $\square$ ст10 二 MSTW 2008 NNPDF 2.1 $\therefore$ HERAPDF 1.5 (b)
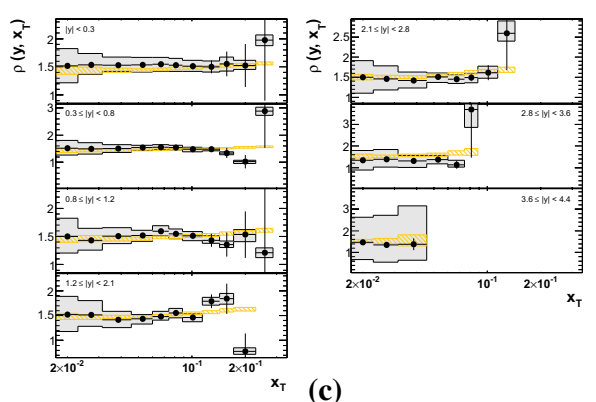

ATLAS

$\int L d t=0.20 \mathrm{pb}^{-1}$

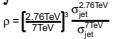
anti- $k_{t} R=0.6$

Data with

statistical
uncertainty
Systematic NLO PQCD $\otimes$
non-pert. corr. non-pert. corr.
(CT10, $\mu=p_{T}^{\text {max }}$ )

Figure 3. (a) Inclusive-jet cross sections as functions of $p_{\mathrm{T}}$ in different regions of $y$ for $R=0.6$. (b) Ratio of data and theory of the cross sections shown in (a). (c) $\rho\left(y, x_{\mathrm{T}}\right)$ as a function of $x_{\mathrm{T}}$ in different regions of $y$. [3]

the proton PDFs [3]. Figure 4a shows the results for the gluon distribution for different cases, HERA data alone in yellow and the results including different sets of ATLAS data are shown with the lines. The impact of the ATLAS data on the PDFs is such that the gluon distribution tends to be harder and the sea distribution tends to be softer when including the ATLAS jet data; the best constraints are provided by the inclusion of both the 2.76 and $7 \mathrm{TeV}$ data sets. Figure $4 \mathrm{~b}$ shows the comparison of the data at $2.76 \mathrm{TeV}$ and the NLO predictions based on the extracted PDFs, given by the red lines: the description of the data is improved, especially in the high $y$ region, which corresponds to high $x$.

\section{Multijet cross sections}

Multijet cross sections provide tests of pQCD directly beyond $O\left(\alpha_{s}^{2}\right)$ and are also sensitive to the value of $\alpha_{s}$. A value of $\alpha_{s}$ was extracted [4] from the ratio of the three-jet and the two-jet cross sections, $N_{3 / 2}\left(p_{\mathrm{T}}^{\text {(all jets) }}\right)=\sum_{i}^{N_{\text {jet }}}\left(d \sigma_{N_{\mathrm{jet}} \geq 3} / d p_{\mathrm{T}, i}^{\mathrm{jet}}\right) / \sum_{i}^{N_{\mathrm{jet}}}\left(d \sigma_{N_{\mathrm{jet}} \geq 2} / d p_{\mathrm{T}, i}^{\mathrm{jet}}\right)$. The measurement is shown in Fig. 5a as a function of $p_{\mathrm{T}}$. The NLO QCD calculations were performed using the program NLOJET++, the scales were set to $\mu_{R}=\mu_{F}=p_{\mathrm{T}}$ and the MSTW2008NLO PDF sets were used; the calculations were corrected for non-perturbative effects. The predictions with $\alpha_{s}\left(M_{Z}\right)=0.110$ and 0.130 are shown in Fig. 5a. The NLO QCD prediction with $\alpha_{s}=0.110$ is closest to the data. A value of $\alpha_{s}\left(M_{Z}\right)$ was extracted from this measurement for $210<p_{\mathrm{T}}<800 \mathrm{GeV}: \alpha_{s}\left(M_{Z}\right)=0.111 \pm 0.006$ (exp. $)_{-0.003}^{+0.016}$ (th.). This value is consistent with the current world average and other experiments. The energy-scale dependence of $\alpha_{s}$ was tested and found to be consistent with the prediction of the renormalisation group equation. Figure $5 \mathrm{~b}$ shows the extracted values by ATLAS, together with the results from other experiments at lower scales. 


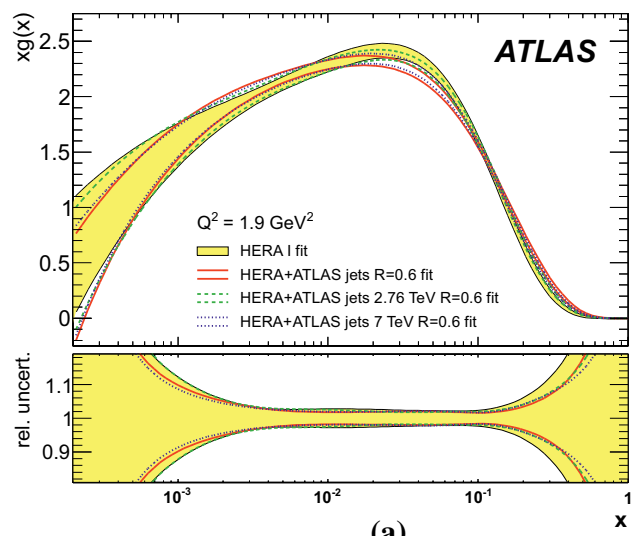

(a)
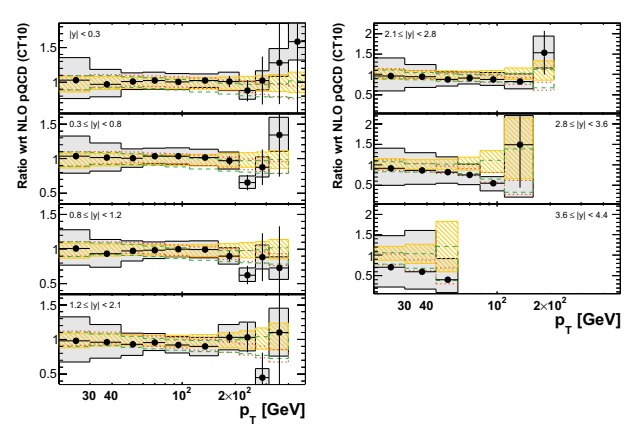

\section{ATLAS}

$\int L \mathrm{dt}=0.20 \mathrm{pb}$ $\sqrt{s}=2.76 \mathrm{TeV}$ Data with
-- statistical
uncertainty $\square \begin{aligned} & \text { Systematic } \\ & \text { uncertainties }\end{aligned}$ NLO PQCD $\otimes$ non-pert. correct

Figure 4. (a) Extracted gluon distribution as a function of $x$. (b) Ratio of data and theory for the inclusive-jet cross sections shown in Fig. 3a. [3]

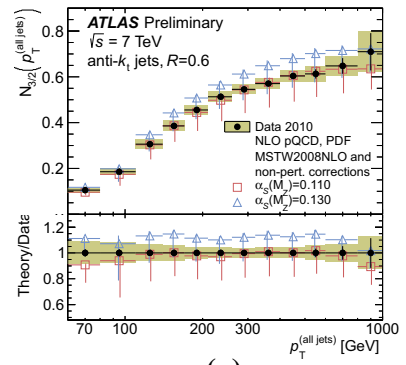

(a)

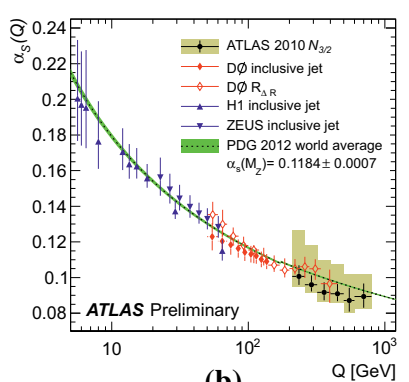

(b)

Figure 5. (a) The $N_{3 / 2}$ ratio. (b) Extracted values of $\alpha_{s}$ from ATLAS (dots); the results from other experiments and the prediction from the renormalisation group equation are also included. [4]

\section{Inclusive isolated photons}

Photons are identified in ATLAS via shower shapes in the calorimeter [5] and required to be isolated to remove the background from neutral-hadron decays into photons. The remaining background is removed via a data-driven method [6]. Photons with transverse energy $E_{T}^{\gamma}>100 \mathrm{GeV}$ and pseudorapidity $\left|\eta^{\gamma}\right|<2.37$, excluding the region $1.52<\left|\eta^{\gamma}\right|<2.37$, were selected for this analysis. Figure 6a shows the cross section as a function of $\left|\eta^{\gamma}\right|$. The measurement is compared to leading-order (LO) parton-shower Monte Carlo and NLO QCD predictions. The LO prediction of PYTHIA gives a good description of the data whereas HerwIG underestimates the normalisation. The NLO QCD prediction of JETPHOX is consistent with the data within the uncertainties. The cross section as a function of $E_{T}^{\gamma}$ was measured in two $\eta^{\gamma}$ regions (see Figs. $6 \mathrm{~b}$ and $6 \mathrm{c}$ ). Values of $E_{T}^{\gamma}$ up to $1 \mathrm{TeV}$ were measured in the central $\eta^{\gamma}$ region. The NLO QCD calculations agree with the data. These cross sections show sensitivity to the proton PDFs: the NLO QCD calculations based on MSTW2008NLO are higher than those based on CT10 and closer to the data at low $E_{T}^{\gamma}$. The theoretical uncertainties due to the PDFs becomes significant at high $E_{T}^{\gamma}$ and so these measurements have the potential to constrain further the PDFs. 


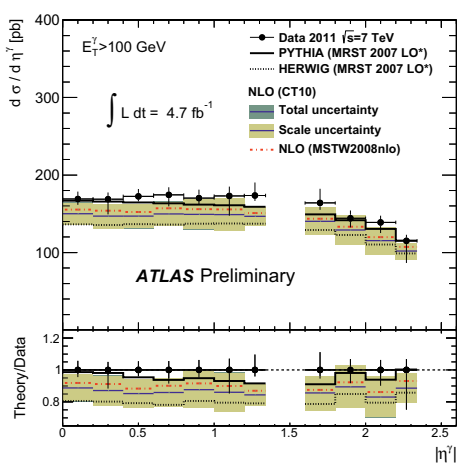

(a)

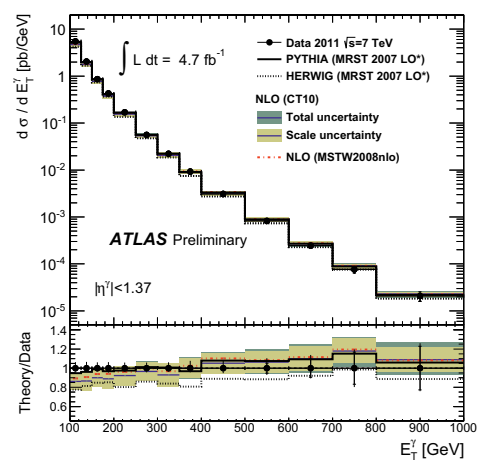

(b)

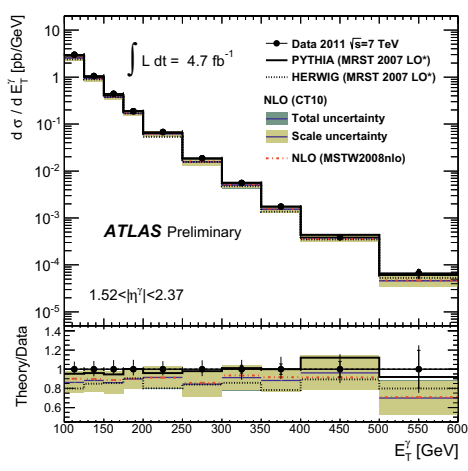

(c)

Figure 6. Isolated-photon cross sections as functions of (a) $\left|\eta^{\gamma}\right|$, (b) $E_{T}^{\gamma}$ for $\left|\eta^{\gamma}\right|<1.37$ and (c) $E_{T}^{\gamma}$ for $1.52<$ $\left|\eta^{\gamma}\right|<2.37$. [6]

\section{Isolated photons in association with jets}

The colour dynamics was tested with the measurements of the cross sections in photon plus jet production [7]. Photons with $E_{T}^{\gamma}>45 \mathrm{GeV}$ and $\left|\eta^{\gamma}\right|<2.37$, excluding the region $1.52<\left|\eta^{\gamma}\right|<2.37$, were selected for this analysis. Jets were identified using the anti- $k_{t}$ algorithm with $R=0.6$ and required to have $p_{\mathrm{T}}>40 \mathrm{GeV}$ and $|y|<2.37$. In this region of phase space, both the experimental and theoretical uncertainties amount to about $10 \%$ and so this study constitutes a stringent test of pQCD. Figure $7 \mathrm{a}$ shows the cross section as a function of the difference in azimuthal angle between the photon and the jet $\left(\Delta \phi^{\gamma \mathrm{j}}\right)$; the predictions of the LO MC models are compared to the data in this figure: PYTHIA and SHERPa give a good description of the data, whereas Herwig fails to describe the data. Figure $7 \mathrm{~b}$ shows the cross section as a function of the invariant mass of the photon-jet system $\left(m^{\gamma \mathrm{j}}\right)$; the NLO QCD calculations of JетPHOX give a good description of this and other differential cross sections studied, except for the cross section as a function of $\Delta \phi^{\gamma \mathrm{j}}$ (see Fig. 7a).

One of the most important variables to test the colour dynamics is $\theta^{\gamma j}$, which is closely related to the scattering angle in the photon-jet centre-of-mass frame and its distribution is sensitive to the spin of the exchanged particle. Figure 8a shows the measured $d \sigma / d\left|\cos \theta^{\gamma \mathrm{j}}\right|$ together with the predictions of the MC models. The predictions for the direct-photon and fragmentation processes are also shown separately; they are predicted to have very different shapes and so this distribution can be used to tune the MC models. To study the colour dynamics, additional requirements must be imposed to remove the bias introduced by the cuts on the transverse momenta and rapidities of the photon and the jet. These additional requirements are: $m^{\gamma \mathrm{j}}>161 \mathrm{GeV}$ and $\left|\eta^{\gamma}+y\right|<2.37$. The measured cross section after applying such requirements (see Fig. 8b) increases as $\left|\cos \theta^{\gamma \mathrm{j}}\right|$ increases, as predicted by QCD. At LO, the two components can be separated and are driven by quark exchange in the case of direct photon and by gluon exchange in the case of fragmentation. The shape of the data is much closer to that of the direct photon (see Fig. 8c) and so this shows the dominance of processes in which a quark is being exchanged in the hard interaction. All these studies demonstrate the validity of the description of the dynamics of isolated-photon production at $O\left(\alpha \alpha_{s}^{2}\right)$. 


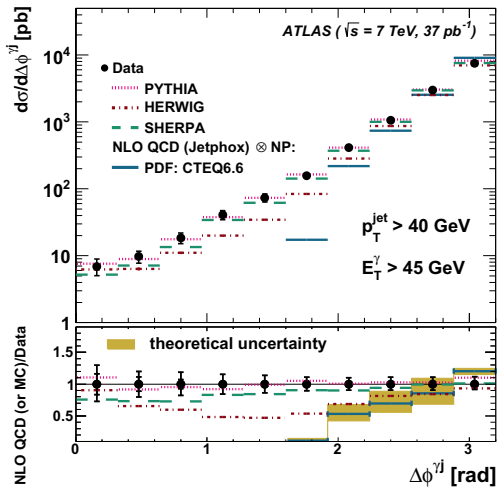

(a)

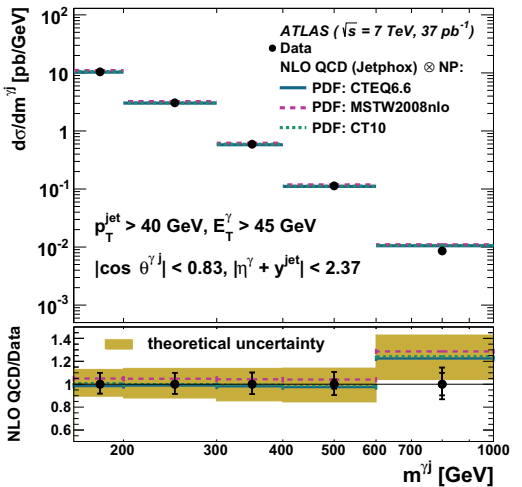

(b)

Figure 7. Isolated-photon plus jet cross sections as functions of (a) $\Delta \phi^{\gamma \mathrm{j}}$ and (b) $m^{\gamma \mathrm{j}}$. [7]

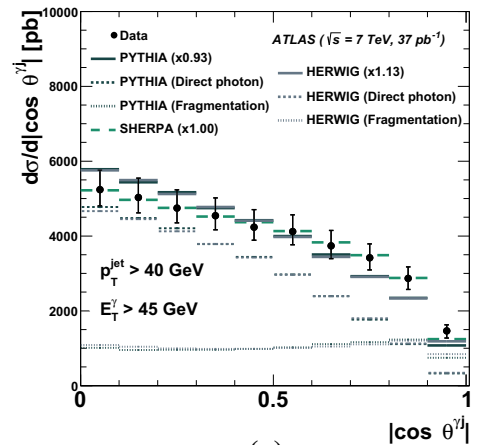

(a)

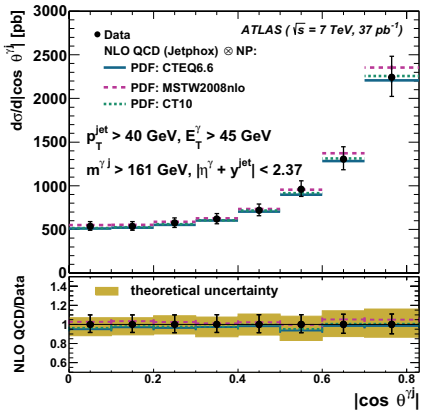

(b)

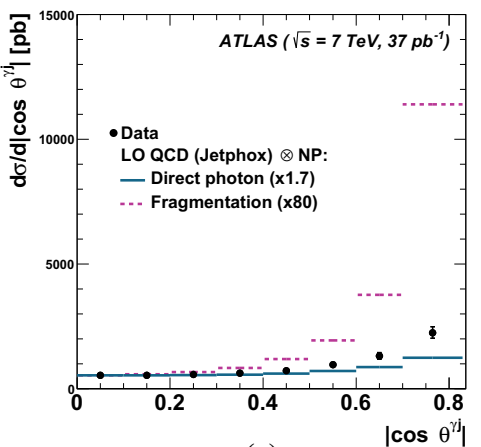

(c)

Figure 8. Isolated-photon plus jet cross sections as a function of $\left|\cos \theta^{\gamma j}\right|$ : (a) without any additional requirements and $(b, c)$ including additional requirements (see text). [7]

\section{Diphoton production}

Diphoton production is the major background to $H \rightarrow \gamma \gamma$. Measurements of cross sections for diphoton production have been performed [8] for the highest- $E_{T}^{\gamma}$ photon with $E_{T}^{\gamma}>25 \mathrm{GeV}$ and the secondhighest- $E_{T}^{\gamma}$ photon with $E_{T}^{\gamma}>22 \mathrm{GeV}$, a phase-space region most relevant for Higgs studies. The two photons were required to be separated by at least 0.4 units in the $\eta-\phi$ plane. Figure 9 shows the differential cross sections for diphoton production as functions of the invariant mass of the two photons $\left(m_{\gamma \gamma}\right)$ and the $p_{\mathrm{T}}$ of the two photons $\left(p_{\mathrm{T}, \gamma \gamma}\right)$. The measurements are compared to the predictions of the LO MC models Pythia and ShERPA. Both models underestimate the normalisation of the data due to the missing higher-order contributions; РүтніA describes the measured $m_{\gamma \gamma}$ distribution better than Sherpa, except at low values, whereas SHERPA gives a good description of the shape of the $p_{\mathrm{T}}$ distribution due to the additional tree-level higher orders.

Figure 10 shows the differential cross sections as functions of $p_{\mathrm{T}, \gamma \gamma}$, difference in azimuthal angle between the two photons $\left(\Delta \phi_{\gamma \gamma}\right)$ and the polar angle of the highest- $E_{T}^{\gamma}$ photon in the Collins-Soper diphoton rest frame $\left(\cos \theta_{\gamma \gamma}^{*}\right)$. In this figure, the data are compared to the following pQCD predic- 

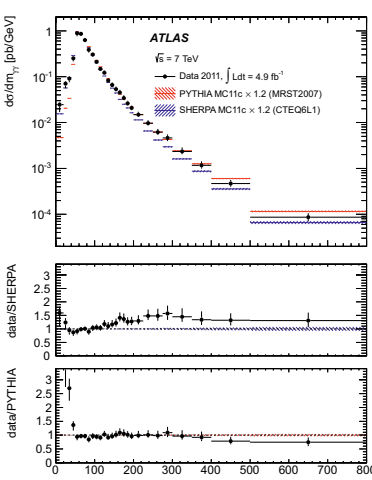

(a)
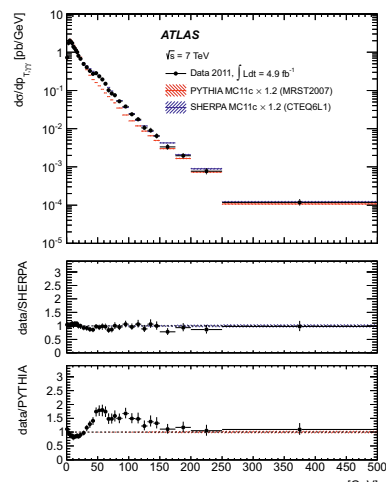

(b)

Figure 9. Differential cross sections for diphoton production as functions of (a) $m_{\gamma \gamma}$ and (b) $p_{\mathrm{T}, \gamma \gamma}$. [8]

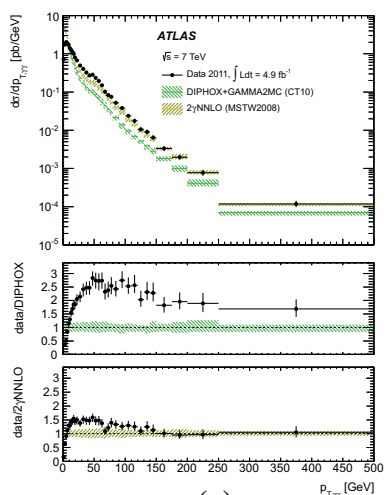

(a)
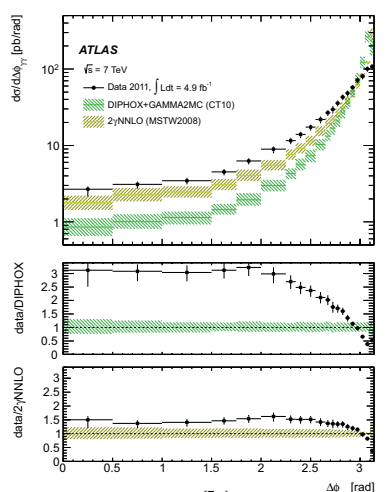

(b)

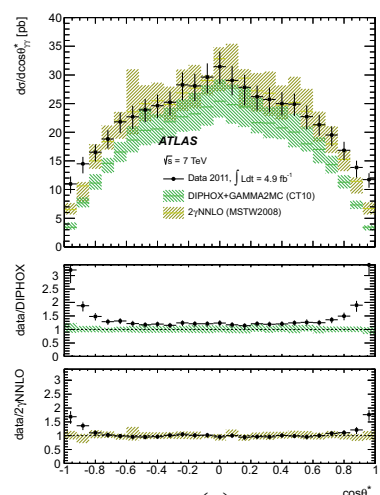

(c)

Figure 10. Differential cross sections for diphoton production as functions of (a) $p_{\mathrm{T}, \gamma \gamma}$, (b) $\Delta \phi_{\gamma \gamma}$ and (c) $\cos \theta_{\gamma \gamma}^{*}$. [8]

tions: $2 \gamma \mathrm{NNLO}$, which contains the contribution of direct photons up to next-to-next-to-leading-order (NNLO), and DiPHox, which includes the NLO contributions of the direct-photon and fragmentation processes and is suplemented with a higher-order correction to the box diagram $g g \rightarrow \gamma \gamma$ using Gamma2mc. The Diphox calculations fail to describe the data, especially because of the missing higher orders. The calculations of $2 \gamma$ NNLO are closer to the data, but still underestimate the data in those regions of phase space in which the fragmentation contribution is more significant. Therefore, improved calculations are needed to understand fully diphoton production.

\section{References}

[1] ATLAS Collaboration, Phys. Rev. D 88, 032004 (2013).

[2] ATLAS Collaboration, New Phys. Jour. 15, 033038 (2013).

[3] ATLAS Collaboration, Eur. Phys. Jour. C 73, 2509 (2013).

[4] ATLAS Collaboration, ATLAS-CONF-2013-041, https://cds.cern.ch/record/1543225. 


\section{ICNFP 2013}

[5] ATLAS Collaboration, JINST 3, S08003 (2008).

[6] ATLAS Collaboration, ATLAS-CONF-2013-022, https://cds.cern.ch/record/1525723.

[7] ATLAS Collaboration, Nucl. Phys. B 875, 483 (2013).

[8] ATLAS Collaboration, JHEP 1301, 086 (2013). 